\title{
Cléopâtre dans le miroir de la peinture du XIXe siècle
}

\author{
Marie-Paule VANLATHEM
}

L'histoire, ainsi que la légende de Cléopâtre, ne sont pas mortes. Plusieurs aspects de sa vie tumultueuse, très courte, ont été peints. Seul les tableaux du 19e siècle sont traités ici. Les auteurs classiques ont été l'une des sources d'inspiration pour évoquer les événements historiques de la dernière reine d'Égypte. Mais aussi l'expédition de Bonaparte au pays des pyramides, dont est issue la Description de l'Égypte, a fortement marqué le siècle qui a suivi, au cours duquel on retrouve des influences égyptiennes dans certaines peintures. Les œuvres décrites ici dans l'ordre chronologique du règne de Cléopâtre se regroupent en quatre parties : son apparition extravagante avec César, sa liaison avec Marc Antoine, sa rencontre avec Octave et sa fin courageuse.

La historia, así como la leyenda de Cleopatra no han muerto. Varios aspectos de su agitada y muy breve vida han sido pintados. En este trabajo sólo vamos a tratar los cuadros del siglo XIX. Los autores clásicos han sido fuente de inspiración para evocar los acontecimientos históricos de la última reina de Egipto. Sin embargo, la Expedición de Bonaparte al país de las pirámides, de la que resultó la Description de l'Égypte, marcó con fuerza la centuria de 1800 , durante la cual encontramos reflejos egipcios en algunos cuadros. Las obras descritas aquí en el orden cronológico del reinado de Cleopatra, son: su forma extravagante de presentarse ante César; la relación con Marco Antonio; el encuentro con Octavio y su valiente final.

KEY WoRDs: Cleopatra, European and North-American painting, XIXth century

$\mathrm{D}^{2}$ u 26 avril au $1^{\text {er }}$ août 2004 s'est tenue au Musée Rath à Genève une grandiose exposition consacrée à "Cléopâtre dans le miroir de l'art occidental". Cet événement s'est accompagné de la publication d'un superbe catalogue auquel plusieurs auteurs ont colla-

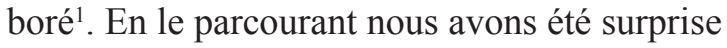
par le nombre de tableaux qui représentent la souveraine lagide à des épisodes marquants de sa courte vie. Les œuvres réunies couvrent une longue période dans laquelle prédominent les $16^{\mathrm{e}}, 17^{\mathrm{e}}$ et $18^{\mathrm{e}}$ siècles. Pour le $19^{\mathrm{e}}$ siècle, moins abondamment représenté, la sélection complète avantageusement les quelques tableaux que $\mathrm{H}$. de Meulenaere a choisis pour illustrer le même thème dans son ouvrage sur l'Égypte ancienne dans la peinture du $19^{\mathrm{e}}$ siècle $^{2}$. Pour l'étude du sujet, cette période est particulièrement intéressante parce qu'elle tranche nettement avec les époques précédentes. En effet, l'expédition du général Bonaparte en Égypte (1798-1802) révèle au monde la richesse archéologique de la terre des pharaons, magnifiquement présentée quelques années plus tard dans les planches de la Description de l'Égypte. À partir de ce moment, des motifs nouveaux s'introduisent occasionnellement dans la peinture en mettant davantage l'accent sur l'origine égyptienne de Cléopâtre et en créant autour d'elle un décor qui rappelle l'antiquité pharaonique. Intéressée

* Je tiens à remercier tout particulièrement le Professeur H. de Meulenaere pour ses précieux conseils. Son savoir m'a guidée dans l'élaboration de ces recherches. Son soutien, son enthousiasme, sa patience et ses encouragements ont modelé le travail que j'offre ici au lecteur. Je reste cependant responsable pour toutes les imperfections qu'il pourrait présenter.

1. Ritschard / Morehead 2004.

2. De Meulenaere 1992. 
par ce phénomène, nous avons, dans les années écoulées, effectué des sondages dans le but d'élargir la documentation rassemblée dans les deux ouvrages précités et de constituer ainsi un dossier aussi complet que possible sur Cléopâtre telle qu'elle est représentée par les peintres du $19^{\mathrm{e}}$ siècle. Nous présentons ci-après le fruit de cette récolte.

Remarquons au préalable que les témoignages des auteurs classiques, en particulier celui de Plutarque, sont l'unique source dont s'inspirent les peintres pour évoquer les avatars de la vie de la dernière reine hellénistique d'Égypte. Ils se concentrent donc sur quelques événements marginaux de son existence mouvementée, très accessoires par rapport à la politique dynastique qu'elle a menée. Nul n'a mieux dessiné le portrait de cette Cléopâtre fantasmatique que Jean Bingen dans une étude récente consacrée à son image 3 .

Notre traitement du sujet présente successivement:

$\neg$ le récit de Plutarque ou d'un autre auteur classique;

- une brève introduction historique;

- la description des tableaux se rapportant au sujet;

$\neg$ une courte énumération des tableaux représentant le même thème, déjà répertoriés dans les publications citées ci-dessus.

Les peintures concernées sont classées dans l'ordre chronologique des événements historiques du règne de Cléopâtre: sa rencontre avec Jules César, sa liaison avec Marc-Antoine, sa soumission à Octave, puis sa mort.

\section{Cléopâtre et Jules César}

Cléopâtre, prenant avec elle un seul de ses amis, le Sicilien Apollodore, monta sur un petit bateau et aborda au palais alors qu'il faisait déjà nuit. N'ayant pas d'autre moyen de passer inaperçue, elle se glissa dans un paquet de couvertures où elle s'étendit de tout son long; Apollodore lia le paquet avec une courroie et le porta à l'intérieur jusqu'à César. On dit que celui-ci se laissa prendre par cette première ruse de Cléopâtre. Il la trouva hardie ; captivé ensuite par sa conversation et sa grâce, il la réconcilia avec son frère, dont il lui fit partager la royauté.

(Plu., Caes. 49. 1-3)

[César] ... mais sa plus grande passion fut pour Cléopâtre: non seulement il lui donna maintes fois des festins qui se prolongeaient jusqu'au jour, mais, l'emmenant avec lui sur un navire pourvu de cabines, il aurait traversé toute l'Égypte et atteint l'Ethiopie, si son armée n'avait pas refusé de le suivre.

(Suet. Iul. LII. 1)

À la poursuite de Pompée après sa victoire à Pharsale, Jules César, âgé alors de cinquante-deux ans, débarqua à Alexandrie en octobre 48 av. J.-C. Cléopâtre, qui n'avait qu'environ vingt ans, l'y rejoignit. Après avoir obtenu de César qu'il la reconnût comme reine légitime d'Égypte, elle le séduisit. Pendant la courte période de leur union en Égypte, au printemps 47 av. J.-C. ${ }^{4}$, ils entreprirent ensemble un voyage sur le Nil, qui n'était probablement pas une simple promenade, mais la réalisation d'une idée de César qui, selon Suétone, désirait atteindre 1'Ethiopie. En même temps, Cléopâtre confirmait sa présence sur le trône et étalait sa fabuleuse richesse.

Ce n'est qu'après le départ de César pour Rome, en juin 47, qu'elle donna naissance à leur fils, Césarion.

\section{Tableaux}

I.1.a. À notre connaissance, Jean-Léon Gérôme

3. Bingen 1996: 235-248.

4. Chauveau 1997: 32; Flamarion 1993: 48 ; Grimal 1998: 277. 
(1824-1904)5 est le seul à avoir immortalisé la rencontre de Cléopâtre et de Jules César. Il est peut-être aussi le premier à avoir peint Cléopâtre dans un décor égyptisant. Sa toile Cléopâtre devant César ${ }^{6}$ montre la reine sortant du tapis, son serviteur agenouillé derrière elle. César est debout près d'elle. Un mur se trouve à droite, un passage à gauche. Cléopâtre, en tenue légère, n'a pas d'attributs pharaoniques. Seule la touche bleue de son collier reproduit un modèle égyptien. César, couronné de lauriers, porte une espèce de chiton blanc, couvert d'un léger manteau rouge. Á côté de ces deux personnes, bien éclairées, se trouve le serviteur habillé d'un rouge foncé rehaussé de vert. Il tient un tapis de couleur foncée qui accentue la pâleur de la reine, tout comme l'architecture sombre du premier plan. La seconde pièce, par contre, semble être illuminée. L'attention du peintre se dirige sur l'architecture, ici pleinement inspirée par l'Égypte ancienne, plutôt que sur les personnages. Ces derniers montrent une certaine négligence : les pieds de Cléopâtre sont amorphes, sa main droite et l'oiseau sur sa tête le sont également. Le bras gauche de César est beaucoup trop long et le valet est presque méconnaissable. On a l'impression d'être en présence d'une étude préparatoire qui annonce le tableau suivant.

I.1.b. Cléopâtre et César ${ }^{7}$, du même peintre, représente aussi la scène précédente. Sortie de ses couvertures, Cléopâtre, debout, est présentée au consul romain par son serviteur. Avec un scarabée ailé suspendu à son collier et un cartouche sur sa poitrine elle est figurée comme une reine égyptienne. L'architecture qui encadre la scène présente des éléments empruntés à l'Égypte ancienne : offrandes aux dieux et textes. Pourrait-on y voir une salle du temple de Deir el-Médineh? ${ }^{8}$ Dans le fond on distingue quatre personnages assis que le catalogue de Sotheby's, New York 23 mai 1990, identifie comme des scribes; observons que César aussi a son calame dans la main et quelques rouleaux de papyrus sur son pupitre.

\section{I.1.c. La galère de Cléopâtre d'Henri-Pierre} Picou (1824-1895) ${ }^{9}$, dont Gauthier a fait une gravure $^{10}$, montre le couple voyageant à bord de la barge de Cléopâtre, luxueux palais flottant fait de cèdre et de cyprès. Le tableau de Picou reflète ce faste. L'œuvre présente un magnifique ensemble d'éléments égyptisants et étrangers. La coiffure de certains personnages, le coffret sur lequel sont étalées des imitations de trouvailles archéologiques, une harpe probablement inspirée de celles de la tombe de Ramsès III, la décoration extérieure du bateau, l'étendard que tient un esclave bronzé dans la main gauche, et l'Horus faucon sur la proue revèlent l'éclat fabuleux de cette croisière exceptionnelle. Le couple royal, assis dans un large fauteuil, se regarde mutuellement. Cléopâtre est drapée de blanc, un diadème d'or sur la tête, César, couronné de lauriers, porte un manteau rouge. Tous les éléments du premier plan correspondent au navire décrit par Plutarque (Vies XIII 25.5) dans lequel Cléopâtre, seule, voyage sur le Cydnus à la rencontre de

5. Né à Vesoul le 11 mai 1824. En 1856 il partit pour l'Égypte avec Léon Belly, Auguste Bartholdi, Frédéric Masson et Emile Augier. Quatre mois sur le Nil pour arriver jusqu'à Assouan, puis quatre mois passés au Caire dans une maison mise à leur disposition par Soliman Pacha (1778-1860). Plusieurs œuvres égyptisantes ont été exposées au Salon de 1857. D'autres séjours en Égypte ont suivi en 1862, 1868, 1869, 1871, 1874, 1880. Le 10 janvier 1904, Gérôme meurt à Paris.

6. 1864-66 ; huile sur papier ; 32 x 39,1 cm ; à Atlanta, voir Lacovara 2001, p .VI ill.: XXIX.

7. 1866 ; huile sur toile ; 1,83 x 1,295 m ; coll. particulière aux États-Unis. Voir s.a. 1981: 74, ill. ; Ackerman 1986: 70 ill.: 218, n 159, qui note " perdu" ; Sotheby’s New York 23 mai 1990, lot 48, ill. ; 183 x 129 cm ; Flamarion 1993: 41, ill.; Humbert 1994: 574, fig. 1.

8. Murat 2003: 23.

9. Né à Nantes le 27 février 1824, mort dans sa ville natale le 17 juillet 1895 .

10. 1875 ; 59,1 x 104 cm ; Bordeaux, Musée Goupil. Voir Flamarion 1993: $48-49$ ill. ; Humbert 1994: 554-555 ill. ; Sartre 1999: 32-34 ill. ; Foreman 2000: 99, 100-101 ill.; Ziegler 1994: 54 ill. 
Marc Antoine (voir ci-dessous). Ici, cependant, la navigation semble se dérouler sur le Nil, devant les montagnes de la chaîne Lybique qu'on aperçoit dans le fond du tableau. La tête couronnée de César plaide elle aussi en faveur d'un voyage sur le Nil.

\section{Tableau déjà répertorié}

I.2.a. Gustave Moreau (1826-1898): Cléopâtre sur le Nil (Ritschard / Morehead 2004, 256-257 ill.).

\section{Cléopâtre et Marc-Antoine}

\section{A. Voyage et rencontre à Tarse sur le Cydnus}

Elle allait se rendre auprès d'Antoine précisément à l'âge où la beauté des femmes est dans tout son éclat et leur esprit dans toute sa force. (...) Elle se mit à remonter le Cydnus sur un navire à la poupe d'or, avec des voiles de pourpre déployées et des rames d'argent manœuvrées au son de la flûte marié à celui des syrinx et des cithares. Elle-même était étendue sous un dais brodé d'or et parée comme les peintres représentent Aphrodite. Des enfants, pareils aux Amours qu'on voit sur les tableaux, debout de chaque côté d'elle, la rafraîchissaient avec des éventails. Pareillement, les plus belles de ses servantes, déguisées en Néréides et en Grâces, étaient les unes au gouvernail, les autres aux cordages.

(Plu., Ant. 25.5 et 26. 1-3)

La liaison de Cléopâtre avec Jules César ne constitue qu'un épisode passager dans la vie de la reine qui ne commence réellement à s'épanouir que lorsqu'elle fait la connaissance de Marc Antoine. Pour sa guerre contre les Parthes, menace permanente sur la frontière orientale de l'empire romain, celui-ci avait un besoin urgent de l'appui militaire et financier de l'Égypte. Despote à la façon orientale, il désirait étendre sa domination à tous les territoires de l'Est de l'empire romain en y incluant l'Égypte. En un mot, soumettre l'Orient à la souveraineté de Rome.

Marc Antoine souhaitait au plus vite rencontrer la reine d'Égypte. Après la mort de Jules César (mars 44), il dépêcha un messager à Alexandrie afin d'inviter Cléopâtre à venir le rejoindre à Tarse en Cilicie où il résidait. $\mathrm{La}$ reine ne se montra pas très pressée et fit attendre le triumvir. Finalement, après plusieurs rappels, elle décida de partir et, pour impressionner le Romain, elle s'embarqua en grande pompe à Alexandrie sur un navire spectaculaire. Après avoir remonté la rivière Cydnus, elle atteignit, à la fin de l'été 41 , Tarse où elle fut accueillie par une grande foule et par Marc Antoine lui-même qui ne tarda pas à céder à son pouvoir de séduction. C'est le début de leur liaison amoureuse.

\section{Tableaux}

II.A.1.a. En avril 1827, Francis Danby (1793-1861) ${ }^{11}$ finissait son tableau The embarkation of Cleopatra on the Cydnus ${ }^{12}$, composition très fantaisiste dont le titre est quelque peu trompeur. Le peintre situe visiblement la scène en Égypte. On y voit, en effet, des statues adossées contre des obélisques, une pyramide dans le lointain, une colonne composite sur laquelle repose une toiture trop lourde. Le décor égyptisant est tout à fait incohérent, et n'a strictement rien à voir avec le Cydnus, fleuve de l'Asie Mineure, né dans le Taurus, qui arrose Tarse pour se jeter dans la Méditerranée, loin de l'Égypte. Cléopâtre, toute petite,

11. Né le 16 novembre 1793 à St. John's près de Killinick en Irlande, mort le 10 février 1861 à Shell House, Exmouth (Angleterre).

12. $1827 ; 60$ x $83 \mathrm{~cm}$; propriétaire actuel inconnu ; vente Sotheby’s Londres 12 juillet $1989, \mathrm{n}^{\circ} 87$. Voir Greenacre 1988 : 73, et p. 102-103 n 27: illustration d'une gravure de E. Goodall d'après la peinture de Danby. 
accompagnée d'un porte-parasol, s'avance dans une brume matinale, où un petit soleil s'infiltre et se reflète dans l'eau, jetant quelque lumière sur les bâtiments navals. La scène se situe visiblement à Alexandrie au moment de l'embarquement de la reine pour Tarse.

II.A.1.b. Le petit tableau de Byam Liston Shaw (1872-1919) ${ }^{13}$, Cleopatra $^{14}$, montre la reine nonchalamment allongée sur des coussins dans un bateau décoré à l'égyptienne. L'étendue d'eau qu'on aperçoit dans le fond, à gauche de la peinture, semble indiquer qu'elle se dirige vers Tarse sur le Cydnus. Sa coiffure est décorée d'un uraeus et sa longue robe, en partie couverte d'un réseau de perles. Devant elle, sont assis deux musiciens qu'elle écoute, les mains relevées derrière la tête.

II.A.1.c. The Arrival of Cleopatra ${ }^{15}$, peint par William Etty (1787-1849) ${ }^{16}$ (fig. 1), montre la jeune reine seule dans son bateau, entourée "d'un essaim de suivantes dénudées jaillissant du navire" 17 sous un joli baldaquin. Sur le toit d'un grand bâtiment de style néo-grec, qui rappelle L'embarquement de Cléopâtre de Jacob van der Ulft (1627-1689) ${ }^{18}$, des curieux découvrent la nouvelle Vénus. Aucun élément égyptien ne figure dans la scène, à l'exception d'une joueuse de harpe agenouillée à la droite de deux danseuses.

II.A.1.d. Un tableau français anonyme, exécuté dans le goût de Claude Le Lorrain (1600-1682), L'embarquement de Cléopâtre à $\operatorname{Tarse}^{19}$ (sic) met l'accent sur le décor : deux grands pavillons, et d'autres plus petits, sont situés dans un cadre architectural. Une faible lumière enveloppe les bâtiments navals dans une profonde perspective. Les personnages minuscules y sont secondaires, tout en se montrant actifs. On ne sait où chercher Cléopâtre.

\section{Tableaux déjà répertoriés}

II.A.2.a. Thomas Buchanan (1822-1872): Cleopatra's Barge (De Meulenaere 1992: 100 ill.).

II.A.2.b. Hans Makart (1840-1884): Kleopatra fährt Markus Antonius auf dem Fluss Kydnus entgegen (Ritschard / Morehead 2004: 346, 348-349 ill.).

II.A.2.c. A. Benini (19e s.): Antoine et Cléopâtre (Ritschard / Morehead 2004: 281 fig. 6).

II.A.2.d. Lawrence Alma-Tadema (1836-1912): The Meeting of Mark Anthony and Cleopatra (Ritschard / Morehead 2004: 360 fig. 3).

\section{B. Banquets}

Il se laissa entraîner par Cléopâtre à Alexandrie, et là, dans des amusements et des jeux de gamin oisif, ... Cléopâtre et lui avaient formé une association dite de la Vie inimitable, et chaque jour ils s'offraient l'un à l'autre des festins en faisant des dépenses incroyables et sans mesure.

(Plu., Ant. 28. 1-2)

13. Né le 13 novembre 1872 à Madras, mort à Londres le 26 janvier 1919.

14. Date inconnue ; huile sur bois ; 30,5 x 25,1 cm ; vente Christie's New York, 30 octobre 2002, $\mathrm{n}^{\circ} 107$ ill.

15. Date inconnue; Walker Art Gallery (Liverpool). Appelé aussi “Triumph of Cleopatra", dans Hughes-Hallett 1990, fig. 16: 241; ou L'arrivée de Cléopâtre en Cilicie dans Bénézit, vol. 5, 1999: 205-206. Voir aussi Hess 1949, sans illustration.

16. Né à York le 10 mars 1787 , et mort dans sa ville natale le 13 novembre 1849 .

17. Grimal 1998: 299, sans ill.; Hughes-Hallett 1990, 241, pl. 16; Humbert 1994: 559 sans ill.

18. Voir le catalogue de vente Ader Picard Tajan, Paris, 19 juin 1981, $\mathrm{n}^{\circ} 43$ ill., 46 x $65 \mathrm{~cm}$, peinture sur bois. Ce tableau est appelé Vue d'un port méditerranéen animé de nombreux personnages, dans le Catalogue de vente de Drouot Richelieu à Paris du 7 octobre 1996, n 5.

19. Première moitié du $19^{\mathrm{e}}$ siècle ; huile sur toile ; 38,5 x 47,5 cm ; vente Ader Picard Tajan Paris, 7 novembre $1990, \mathrm{n}^{\circ} 437$ ill. 
Il y avait deux perles, les plus grosses qui eussent jamais existé, l'une et l'autre propriété de Cléopâtre, dernière reine d'Égypte ; elles les avait héritées des rois de l'Orient. Au temps où Antoine se gavait journellement de mets choisis, Cléopâtre, avec le dédain à la fois hautain et provocant d'une courtisane couronnée, dénigrait toute la somptuosité de ces apprêts. Il lui demanda ce qui pouvait être ajouté à la magnificence de sa table; elle répondit qu'en un seul dîner elle engloutirait dix millions de sesterces. Antoine était désireux d'apprendre comment, sans croire la chose possible. Ils firent donc un pari ; le lendemain, jour de la décision, elle fit servir à Antoine un dîner d'ailleurs somptueux - il ne fallait pas que ce jour fût perdu -, mais ordinaire. Antoine se moquait et demandait le compte des dépenses. Ce n'était, assura-t-elle, qu'un à-côté, le dîner coûterait le prix fixé, et seule elle mangerait les dix millions de sesterces. Elle ordonna d'apporter le second service. Suivant ses instructions, les serviteurs ne placèrent devant elle qu'un vase, empli d'un vinaigre dont la violente acidité dissout les perles. Elle portait à ses oreilles les bijoux extraordinaires, ce chef-d'œuvre de la nature vraiment unique. Alors qu'Antoine se demandait ce qu'elle allait faire, elle détacha l'une des perles, la plongea dans le liquide, et, lorsqu'elle fut dissoute, l'avala. Elle se disposait à engloutir l'autre de la même façon; L. Plancus, arbitre de ce pari, mit le holà et prononça qu'Antoine était vaincu, présage qui s'est accompli.

(Plin. HNIX, 119-121)

Après un bref séjour à Tarse, le couple arriva au début de l'hiver 41 à Alexandrie. Cléopâ-

[134 ] tre et Marc Antoine y passèrent quelques mois ensemble, menant une vie d'un luxe effréné qu'illustrent en particulier les somptueux banquets qu'ils organisèrent. Leur idylle fut interrompue au début de 40 lorsque le triumvir, apprenant l'invasion des Parthes en Syrie, alla prendre la tête de son armée, quittant sa favorite pour presque quatre années. C'est en automne de la même année que celle-ci accoucha de jumeaux, un garçon et une fille, avec lesquels elle partit en 37 à Antioche afin de rejoindre le Romain.

\section{Tableau}

II.B. 1.a. La toile d'Ange François (1800-1867) ${ }^{20}$ Antoine et Cléopâtre ${ }^{21}$ se rapporte à un évènement raconté par Pline l'Ancien : l'histoire de la perle. Cinq personnes, regardant chacune dans une direction différente, feignent de se désintéresser de Cléopâtre qui va dissoudre sa précieuse perle dans une coupe de vinaigre. Le pari de la perle, contrôlé par le juge Livius Plancus, représenté probablement par le vieil homme à gauche de Marc Antoine, démontre une fois de plus les richesses de la reine d'Égypte. Il n'y a point de banquet, point de plats ou friandises, seule une couronne de fleurs garnit la table ronde. Le centre du tableau est formé par les deux personnages principaux; multicolores, ils se détachent sur les tons plus sombres du fond. Une lumière, apparemment venant d'en haut, tombe sur le crâne du juge et sur un personnage vêtu d'un némès, seule présence égyptienne dans la peinture. Un valet noir, habillé d'un vêtement foncé rehaussé de rouge, fait face à l’Égyptien.

\section{Mort et obsèques de Marc Antoine}

Elle-même avait fait construire près du temple d'Isis des caveaux et des tombeaux d'une beauté et d'une hauteur extraordinaires.

$$
\text { (Plu., Ant. 74. 2) }
$$

Elle se réfugia dans son mausolée et fit baisser les herses fortement assujetties par des serrures et des verrous, puis elle envoya dire à Antoine qu'elle était morte. Il le crut.

(Plu., Ant. 76. 4)

20. Né à Bruxelles le 2 janvier 1800, mort dans sa ville natale en 1867 (Berko 1981: 291).

21. Date inconnue ; huile sur toile ; 43 x $55 \mathrm{~cm}$; propriétaire actuel inconnu ; ventes: Drouot Paris, 29 juin $1988, \mathrm{n}^{\circ} 95$; Christie's Monaco, 7 décembre 1990, n 355 ill. 
Il se frappa alors au ventre et se laissa choir sur son lit. Mais le coup ne causa pas immédiatement la mort: le sang s'arrêta de couler dès qu'Antoine fut étendu; il revint à lui et supplia ceux qui étaient là de l'égorger. Ils s'enfuirent de la chambre, où il cria et se débattit jusqu'à l'arrivée de Diomède, secrétaire de Cléopâtre, qu'elle avait chargé de le porter auprès d'elle dans le mausolée.

(Plu., Ant. 76. 9-11)

Ayant donc appris qu'elle vivait, Antoine pressa vivement ses serviteurs de le prendre dans leurs bras, et ils le portèrent à l'entrée du

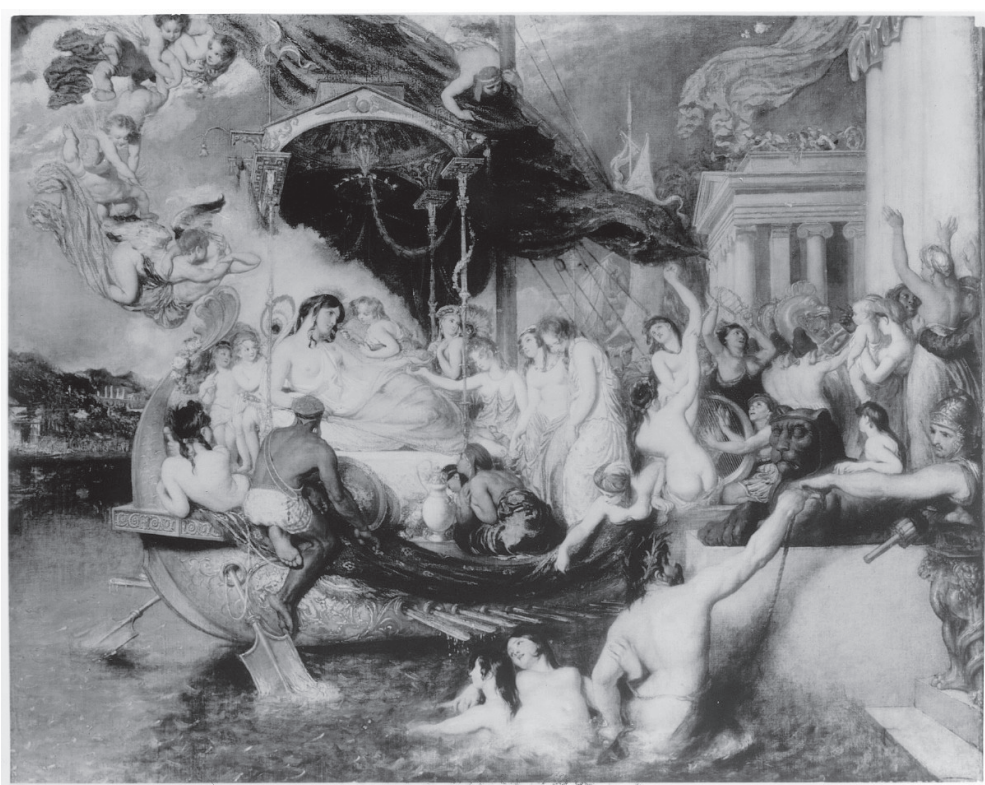

(C) National Museums and Galleries on Merseyside, Liverpool.

Figure 1

l'enleva pas à Cléopâtre; elle l'ensevelit de ses propres mains avec une magnificence royale, et elle put disposer à cette fin de tout ce qu 'elle voulut.

(Plu., Ant. 82. 2)

Elle demanda d'abord à César la permission d'offrir des libations à Antoine. Il la lui accorda. Alors elle se fit porter au tombeau et, se prosterna auprès du tertre funéraire avec ses suivantes ordinaires.

(Plu., Ant. 84. 3)

déchira ses propres vêtements pour l'en couvrir et, se frappant la poitrine et la meurtrissant de ses mains, elle essuya le sang avec son visage, en l'appelant son maître, son époux, son Impérator, et elle oubliait presque ses maux à elle dans sa pitié pour ceux d'Antoine. Celui-ci arrêta ses lamentations et demanda du vin à boire, soit qu'il eût soif, soit qu'il espérât être ainsi délivré plus promptement de la vie.

(Plu., Ant. 77. 5-6)

Quant au corps d'Antoine, bien qu'il fût réclamé par plusieurs rois et généraux qui voulaient lui faire des funérailles, César ne
$\mathrm{Au}$ cours d'une cérémonie fastueuse à Alexandrie en 34, Cléopâtre et ses enfants reçurent d'Antoine des donations ${ }^{22}$ importantes de territoires conquis qui révoltèrent l'opinion romaine et en particulier son collègue triumvir Octave qui, en outre, ne pardonna pas à Marc Antoine d'avoir répudié sa sœur Octavie qu'il avait épousée en 40. De plus, celui-ci, dans son testament ${ }^{23}$, demanda à sa mort d'être enterré en Égypte au côté de Cléopâtre. Faute inexcu-

22. Dobias 1934: 287-314 ; Flamarion 1993: 72-73 ; Chauveau 1998: 87-88, 89.

23. Selon la coutume déposé au temple des Vestales. Octave, pas toujours très scrupuleux, a fait saisir le texte, et en donna lecture au Sénat. Voir von Wertheimer 1935: 265 ; Daix 1960: 243, 245 ; Flamarion 1993: 79-80 ; Foss 1997: 145; Chauveau 1998: 94; Foreman 2000: 140-41. 
sable qui fit grand scandale à Rome. Devenus ennemis, les Romains s'affrontèrent dans une bataille navale près d'Actium (2 septembre 31$)$. Cléopâtre, qui assista dans son navire à la débâcle qui en résulta, prit honteusement la fuite, suivie par Marc Antoine. Il leur restait encore onze mois à vivre. Antoine, déprimé, songeait à se suicider, mais quelques amis l'en empêchèrent et lui conseillèrent de prendre la direction d'Alexandrie. De triste humeur, il se réfugia tout près de l'île de Pharos ${ }^{24}$. Lentement, il reprit moral, et leur "vie inimitable" recommença, le couple se transformant bientôt en "ceux qui vont mourir ensemble" 25 . Au printemps 30, Octave débarqua à Péluse et se dirigea sur Alexandrie, pour y arriver au début de l'été ${ }^{6}$. Cléopâtre avait ordonné à ses troupes de ne lui opposer aucune résistance. Le $1^{\text {er }}$ août 30 , Marc Antoine se lança de nouveau contre Octave. C'était une vaine tentative, dans laquelle il perdit des hommes passés à l'ennemi ${ }^{27}$. En désespoir de cause, se croyant trahi par son épouse qui cherchait à intriguer auprès d'Octave, et abusé par la (fausse) nouvelle de sa mort, Marc Antoine se suicida le $1^{\text {er }}$ août 30 .

\section{Tableaux}

II.C.1.a. Dans son tableau Antonius dodelijk verwond bij Cleopatra ${ }^{28}$, Louis Moritz $(1773-1850)^{29}$ montre Marc Antoine mourant, sans qu'aucune blessure ou goutte de sang ne se remarque sur son corps. N'ayant point l'allure d'un Romain, il est couché sur [136] une espèce de lit, soutenu du côté de la tête par un sphinx ailé. Au pied, on distingue va- guement une grande tête d'animal, inspirée des lions égyptiens de la Cordonata du Capitole à Rome ${ }^{30}$. Epuisé, il pose sa main droite sur le poignet de Cléopâtre qui reste là, assise, très pâle, immobile, sans même regarder son époux. Couronnée d'un diadème à petites pointes, et vêtue de façon ni spécifiquement grecque ni spécifiquement romaine, à l'exception de ses sandales, elle est affaissée sur le bord d'une chaise de type romain. Les servantes de Cléopâtre ressemblent davantage à des Hollandaises qu'à des Romaines ou des Égyptiennes. L'une d'elles se penche sur Marc Antoine, l'autre, levant sa main droite au front, lui offre le vin qu'il a demandé. À gauche se trouve un guéridon, reposant sur un sphinx ailé à longues pattes, dont la tablette supporte un vase et un collier de perles. Derrière la chaise de Cléopâtre, par terre, on aperçoit le costume militaire du Romain. Le petit espace où se déroule ce drame se situe dans un décor égyptien. Dans le fond à gauche se dessinent les chapiteaux de colonnes papyriformes fermés dans le style du Nouvel Empire. À droite, une porte égyptienne mène à une pièce au fond de laquelle on distingue la façade d'une construction égyptienne qui rappelle un bâtiment de style gréco-romain, montrant un chapiteau à tête d'Hathor. Contre le mur du passage on croit distinguer un grand vase canope.

\section{II.C.1.b. Dans The Death of Mark Anthony ${ }^{31}$ de Charles Elder (1821-1857)32, daté 1847, le Romain est couché sur un lit, une peau de panthère sous les genoux. Cléopâtre se pen- che sur lui et essuie son sang. Ils se regardent, conscients de ce qui va se passer. Antoine a ré-}

24. Huss 2001: 745, 746.

25. Daix 1960: 266-267.

26. Flamarion 1993: 91.

27. Huss 2001: 748.

28. 1823-1825 ; huile sur toile ; 134 x 263 cm ; Amsterdam, Rijksmuseum Inv. SK-A-1084.

29. Né à La Haye le 29 octobre 1773, mort à Amsterdam le 22 novembre 1850.

30. Humbert 1989: 14.

31. Huile sur toile, 145 x 190,5 cm ; vente Sotheby's Londres, 27 mai 1987, nº 301 ill.

32. Né en 1821, mort à Londres en 1851. 
clamé du vin qu'une servante lui apporte dans un gobelet. L'équipement militaire de Marc Antoine, un beau casque, est jeté par terre. Un archer, flèches sur le dos, un Arabe, à la peau foncée et portant un turban sur la tête, et une servante de l'autre côté du lit sont seuls à assister à cette scène. Les premiers se contentent de regarder, dans une attitude peu respectueuse. Une petite ouverture en haut, à droite, laisse entrevoir les nuages du ciel, donnant ainsi l'impression de se trouver dans le mausolée de Cléopâtre où bientôt elle-même va se suicider.

II.C.1.c. "Tes ennemis me destinent à orner leur triomphe: on va pour jamais m'éloigner de toi, et les larmes que je répands sur ton tombeau sont les dernières que j'y verserai". Tel est le texte du catalogue de Sotheby's qui illustre le tableau Cléopâtre reine d'Égypte au tombeau d'Antoine ${ }^{33}$, de Louis Marie Baader (1828-1919) $)^{34}$, exposé au Salon de 1895 à Paris. Cléopâtre, couchée par terre dans une attitude hystérique, lève ses bras vers le haut monument d'Antoine. Deux servantes sont assises dans le fond, regardant la souffrance de leur reine. Une d'elles, accroupie, repose sa tête sur les genoux de l'autre. Le décor opulent de cette œuvre contribue à sa beauté. Tout rappelle l'antiquité égyptienne. Avec sa corniche ornée d'un soleil ailé entouré de deux cobras, son tore, ses frises aux cobras, ses figurines humaines, le caveau, un peu fantaisiste, tend à imiter un tombeau égyptien. Cléopâtre l'a couronné d'une guirlande de fleurs et quelques roses sont dispersées sur les marches et sur le sol à côté d'une corbeille. Un chasse-mouches en forme de papyrus déployé en éventail gît sur le tapis. La construction qui flanque le tombeau est décorée d'une tête d'Hathor. Le fond de la pièce est formé par une colonne insérée dans une paroi portant des éléments floraux et une scène d'adoration. Comme dans certaines autres peintures plus anciennes $^{35}$, une grande statue romaine, dressée devant la paroi, évoque le souvenir de Jules César. La couleur du grès, prédominante dans le tableau, est adoucie par des tons de rose, de bordeaux, de vert et d'orange.

\section{Tableau déjà répertorié}

II.C.2.a. Eugène Ernest Hillemacher (1818-1887): Antoine mourant porté vers Cléopâtre (Ritschard / Morehead 2004: 336-337 ill.).

\section{Les derniers JOURS de Cléopâtre}

\section{A. Cléopâtre et Octave Auguste}

Il [Marc Antoine] était sur le point d'expirer lorsque Proculeius se présenta de la part de César. En effet, aussitôt qu' Antoine, après s'être frappé, avait été porté auprès de Cléopâtre, un de ses gardes, nommé Dercetaeus avait pris son épée, l'avait cachée sous son vêtement, était sorti secrètement et, courant vers César, lui avait annoncé le premier la mort d'Antoine, en lui montrant son épée ensanglantée.

(Plu., Ant. 78. 1)

Ensuite il [César] envoya Proculeius en lui ordonnant avant tout de prendre, s'il le pouvait, Cléopâtre vivante, car il craignait pour ses trésors, et il pensait que sa présence contribuerait grandement à l'éclat de son triomphe. Cependant elle refusa de se mettre entre les mains de Proculeius. Pour lui parler, il s'approcha du mausolée, mais resta dehors à la porte qui était de plain-pied avec le sol et se trouvait solidement fermée tout en laissant un passage à la voix.

(Plu., Ant. 78. 4-5)

Quand Proculeius eut examiné les lieux et fait son rapport à César, celui-ci envoya Gallus en vue d'un nouvel entretien avec Cléopâtre.

33. Date inconnue ; huile sur toile ; 89 x $124 \mathrm{~cm}$; Sotheby’s Londres, 24 octobre $1996, \mathrm{n}^{\circ} 347$ ill.

34. Né à Lannion (Côtes-du-Nord) en 1828, mort à Paris en 1919.

35. Batoni: Ritschard / Morehead 2004: 229-231 (Dijon) ; Mengs: Humbert 1994: 569 (Augsbourg); Gauffier: Humbert 1994: 571 (Édimbourg). 
Gallus vint à sa porte et, à dessein, fit traîner en longueur la conversation. Pendant ce temps, Proculeius appliqua une échelle au mur et entra par la fenêtre par laquelle les femmes avaient introduit Antoine, et il descendit aussitôt, accompagné de deux satellites, à la porte même où se tenait Cléopâtre occupée à écouter Gallus. L'une des deux femmes enfermées avec Cléopâtre ayant crié: "Malheureuse Cléopâtre, te voilà prise!", elle se retourna et, apercevant Proculeius, elle tenta de se frapper avec une dague de brigand qu'elle se trouvait porter à sa ceinture, mais Proculeius, courant vite à elle et l'entourant de ses bras, dit: “Tu fais tort, Cléopâtre, à toi-même et à César, que tu veux priver d'une grande occasion de montrer sa bonté, et ainsi tu calomnies le plus doux des souverains comme s'il était sans foi et sans pitié". En même temps il lui enleva son arme et secoua son vêtement dans la crainte qu'elle n'y cachât quelque poison. César envoya ensuite un de ses affranchis, Épaphrodite, avec ordre de la garder vivante et de veiller rigoureusement sur elle, en lui accordant d'ailleurs tout ce qui pouvait lui procurer la vie plus facile et la plus agréable.

(Plu., Ant. 79. 1-6)

Après la mort de Marc Antoine, la principale préoccupation d'Octave était de s'emparer de Cléopâtre vivante pour l'emmener triomphalement à Rome avec ses enfants. C'est la raison pour laquelle il fit veiller sur elle par peur qu'elle ne se suicide.

\section{Tableaux}

III.A.1.a. Le Rijksmuseum d'Amsterdam possède le tableau de Joannes Echarius Carolus Alberti (1777-1850) ${ }^{36}$ Proculeius weerhoudt Cleopatra ervan zich te doorsteken ${ }^{37}$. Plus que les autres artistes, ce peintre a fidèlement illustré le texte de Plutarque, en observant, quelques libertés mises à part, tous les détails qu'il fournit: l'échelle, les deux satellites, la dague de brigand dans la main de Cléopâtre, Proculeius l'entourant de ses bras. Dans le fond on voit Marc Antoine, mort sur un lit, le bras droit pendant. La petite lampe qui illumine son cadavre est empruntée aux fresques de Pompéi et est également présente dans $L a$ Mort de Cléopâtre de Boulanger de Boisfremont (1828) ${ }^{38}$. À droite, deux servantes interpellent Cléopâtre. La ligne oblique à gauche, qui dessine l'extérieur du bâtiment, imite une architecture égyptienne.

III.A.1.b. L'arresto di Cleopatra ad Alessandria d'Egitto ${ }^{39}$, magnifique petit tableau de Giovanni Muzzioli (1854-1894) ${ }^{40}$ (fig. 2), présente un cadre égyptisant dont l'architecture forme l'élément principal. Le pylône est décoré d'un dieu Ré debout en face de la déesse Hathor, à tête de vache, assise. Les colonnes hathoriques dans le fond rappellent la salle hypostyle du temple d'Hathor à Dendera, dessiné par Denon et peint par David Roberts en 1841 à un moment où le temple était encore en grande partie ensablé. Un linteau au-dessus montre un soleil ailé, flanqué de deux cobras et, plus haut, deux rangées de hiéroglyphes fantaisistes. Muzzioli invente un escalier pour accéder au temple. La délégation romaine, des lances et une bannière SPQR dans les mains, s'est groupée à gauche devant les murs d'entrecolonnement; à droite des curieux se sont rassemblés tout près du mur. Au rez-dechaussée, adossés à un pylône, on remarque trois personnages dont un Égyptien agenouillé qui regarde sa reine. Un grand signe ankh décorant le pylône contraste avec l'atmosphère macabre qui remplit l'espace. En haut de l'escalier, à la sortie du temple, Cléopâtre for-

36. Né à Maastricht le 21 juin 1777, mort vers 1850 .

37. 1810 ; huile sur toile ; 122 x $160 \mathrm{~cm}$; Amsterdam Rijksmuseum, Inv. SK-A-641.

38. Ritschard / Morehead 2004: 328, 329 ill.

39. Date inconnue ; huile sur toile ; 64 x 39 cm ; Collection privée. Voir Juler 1987: 183 ill.

40. Né à Modena le 10 février 1854, mort dans sa ville natale le 5 août 1894. 


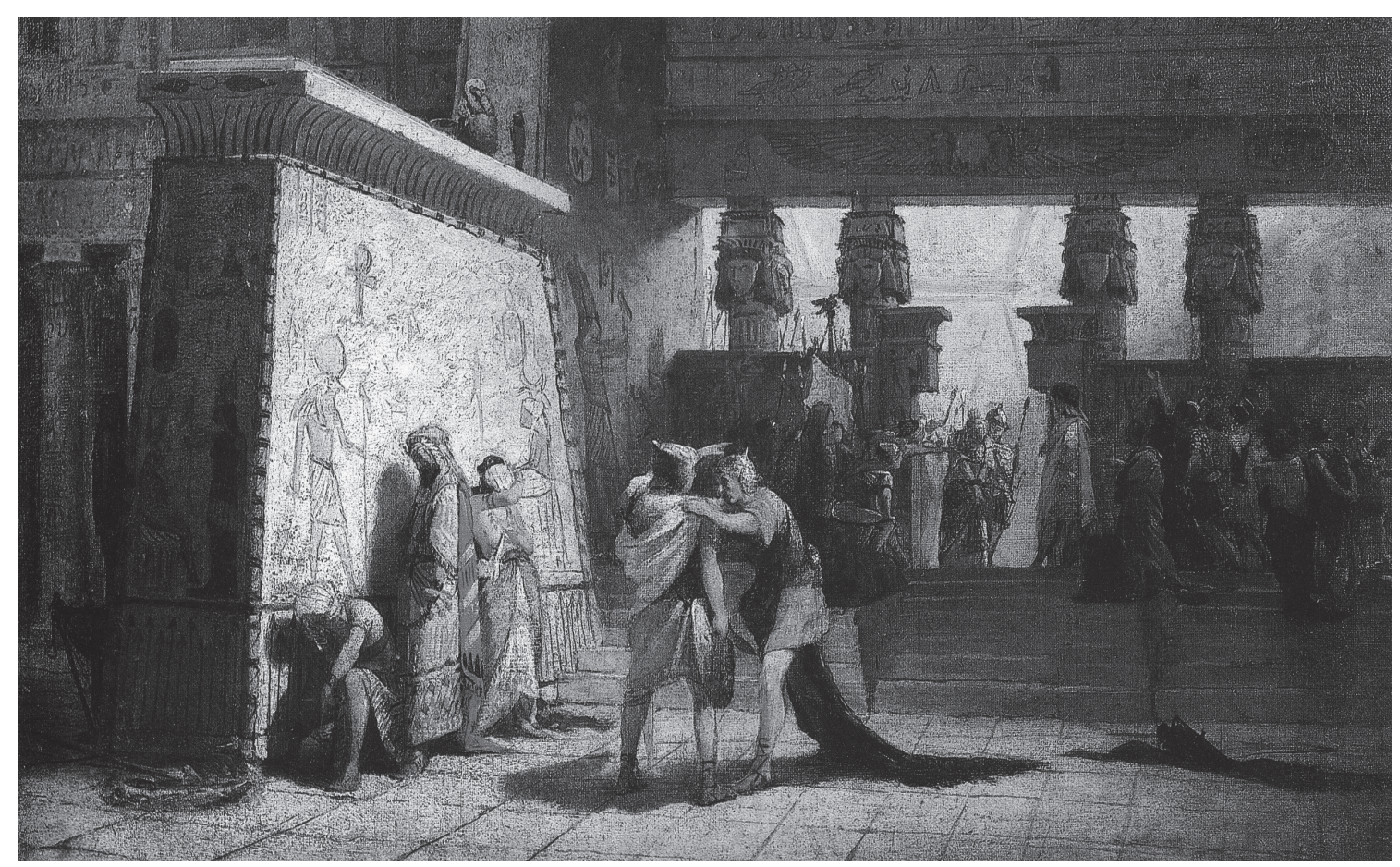

(c) ACR Edition internationale Courbevoie, Paris.

Figure 2

tement illuminée, est arrêtée par des Romains. L'un d'eux, Proculeius (?), tient une lance dans la main gauche. Cléopâtre a les mains ligotées, et sa tête, coiffée de la dépouille de vautour, est inclinée.

\section{B. Préparatifs du suicide}

[Après la bataille près d'Actium] "Cependant Cléopâtre rassemblait toute sorte de poisons mortels et, pour savoir lequel était le moins douloureux, elle les faisait prendre à des prisonniers condamnés à mort. Quand elle se fut aperçue que ceux dont l'effet est prompt causent une mort non moins douloureuse que rapide, et que les poisons plus doux n'agissent que lentement, elle essaya le venin des serpents ; elle en fit appliquer, sous ses yeux, de différentes espèces à divers individus, et elle renouvelait chaque jour ces expériences. Elle ne trouva guère que la morsure de l'aspic, entre toutes, pour amener sans convulsion ni gémissement à une sorte de sommeil et d'engourdissement, accompagné d'une sueur légère au visage et d'un affaiblissement des sensations ...

(Plu., Ant. 71. 6-8)

Le 9 août 30, Cornelius Dolabella, jeune ami de César, ayant quelques sympathies pour la reine, l'avertit des intentions d'Octave Auguste qui souhaitait l'emmener avec ses enfants comme prisonniers, sur le chemin de son retour à Rome. Cléopâtre repoussa catégoriquement cette humiliation et préféra préparer son suicide.

\section{Tableaux}

III.B.1.a. Suzanne Daynes-Grassot-Solin ${ }^{41}$ peint Cléopâtre essayant les poisons sur les escla-

41. Née en 1881 ou 1884 à Paris. La date de sa mort est inconnue. Artiste qui s'est spécialisée dans la représentation de danseuses, d'intimités féminines, de nus. Voir Bénézit, vol. 4, 1999: 311. 
$v e s^{42}$. Dans une atmosphère de brume matinale, froide, Cléopâtre allongée nonchalamment et s'appuyant sur son coude, observe, d'un regard intéressé, un peu à distance, les esclaves se tordant de souffrance à la suite du poison qu'elles ont avalé, et qu'une servante, élégamment habillée, un némès sur la tête, distribue encore. Un petit esclave noir, portant un éventail, est assis aux pieds de Cléopâtre. A côté de lui, devant l'entrée, un gardien, coiffé du némès, porte une lance. Dans le fond, derrière Cléopâtre, figurent des arbres ou de grands bouquets de lotus, simulant un jardin ou une terrasse, et un soleil décoré d'un vautour (?) ailé. La décoration des murs du lieu où se passe la scène, est inspirée par des représentations du Nouvel Empire, comme le cheval sur la paroi gauche, portant des plumes d'autruche sur la tête. Une lumière tombant d'en haut divise la toile diagonalement, laissant Cléopâtre à l'abri des rayons.

\section{Tableaux déjà répertoriés}

III.B.2.a. Alexandre Cabanel (1823-1889): Cléopâtre (Ritschard / Morehead 2004: 245 fig. 1).

III.B.2.b. Alexandre Cabanel (1823-1889): Cléopâtre essayant des poisons sur des condamnés à mort (De Meulenaere 1992: 99 ill.; Ritschard / Morehead 2004: 261 ill.).

III.B.2.c. Gustave Moreau (1826-1898): Cléopâtre essayant du poison sur les esclaves (Rits[ 140 ] chard / Morehead 2004: 354, 355 ill.).

\section{Le suicide de Cléopâtre}

Elle couronna de fleurs et embrassa la tombe, puis elle se fit préparer un bain. Une fois baignée, elle se mit à table et prit un repas somptueux. Un homme arriva alors de la campagne, portant un panier. Comme les gardes lui demandaient ce qu'il contenait, il l'ouvrit, écarta les feuilles et leur montra qu'il était plein de figues. Les gardes admirent la beauté et la grosseur des fruits, l'homme sourit et les invita à en prendre; ainsi mis en confiance, ils le laissèrent entrer avec ce qu'il portait. Après son déjeuner, Cléopâtre prit une tablette qu'elle avait écrite et cachetée, et l'envoya à César, puis, ayant fait sortir tout le monde, à l'exception de ses deux femmes dont j'ai parlé, elle ferma la porte. Quand César eut décacheté la tablette et lu les prières et les supplications par lesquelles elle lui demandait de l'ensevelir avec Antoine, il comprit aussitôt ce qu'elle avait fait. ... il envoya en toute hâte des gens pour voir ce qui s'était passé. Le drame a été rapide; car, venus en courant, ils surprirent les gardes qui ne s'étaient aperçus de rien, et, ouvrant la porte, ils trouvèrent Cléopâtre morte, couchée sur un lit d'or et vêtue de ses habits royaux. L'une de ses suivantes, appelée Iras, expirait à ses pieds; l'autre, Charmion, déjà chancelante et appesantie, arrangeait le diadème autour de la tête de la reine.

(Plu., Ant. 85. 1-7)

L'aspic, dit-on, fut apporté à Cléopâtre avec ces figues et il avait été caché sous les feuilles, car elle l'avait ainsi ordonné, afin que l'animal l'attaquât sans même qu'elle le sût ; mais, en enlevant des figues, elle le vit et dit: "Le voilà donc", puis elle dénuda son bras et l'offrit à la morsure ... Mais personne ne sait la vérité, car on a dit aussi qu'elle portait toujours du poison dans une épingle à cheveux creuse et qu'elle cachait cette épingle dans sa chevelure.

$$
\text { (Plu., Ant. 86. 1) }
$$

Il [Auguste] désirait si vivement la réserver à son triomphe, qu'il fit même venir des psylles pour sucer le venin de sa plaie, car on croyait qu'elle s'était tuée en se faisant mordre par un aspic.

(Suet. Aug. XVII. 8)

Personne ne sait au juste comment elle périt ... quelques-uns rapportent qu'elle y appliqua un aspic qui lui avait été apporté soit dans une aiguière,

42. Voir Flamarion 1993: 137, 156, n ${ }^{\circ} 137$ qui ne fournit point de détails sur ce tableau. 
soit parmi des fleurs, ... César, à la nouvelle de la mort de Cléopâtre, fut frappé de douleur ; il visita son corps, fit venir des remèdes et des psylles pour tâcher de la sauver ... César, n'ayant pu par aucun moyen rappeler Cléopâtre à la vie ...

(Dion Cassius VII, LI. 14)

Peu de temps après le suicide et les funérailles de Marc Antoine qu'elle organisa avec l'accord d'Octave, Cléopâtre, comprenant qu'elle ne séduirait pas le vainqueur d'Actium et craignant que celui-ci ne l'emmène enchaînée à Rome pour son retour triomphal, se suicida à son tour le 10 août 30 . Les informations que nous possédons sur sa mort reposent en grande partie sur le témoignage de Plutarque. Même aujourd'hui, les circonstances de sa fin tragique demeurent entourées de mystère.

Les poètes latins, Virgile, Horace, Properce ${ }^{43}$, peu élogieux à l'égard de Cléopâtre ${ }^{44}$, parlent, de plus, d'un cobra ${ }^{45}$. Plus tard, au tournant du siècle, le géographe Strabon note prudemment deux versions : le poison et la morsure de serpent. La version romaine ${ }^{46}$, vraie ou imaginaire, est peut-être basée sur le fait qu'Auguste fit "venir des psylles pour sucer le venin de sa plaie", ou sur la statue de Cléopâtre, un cobra autour du bras, qui remplaça la reine morte dans le triomphe d'Octave à Rome ${ }^{47}$. Quoi qu'il en soit, toujours est-il que la morsure de serpent a le plus souvent inspiré les génies des arts.

\section{Tableaux}

III.C.1.a. La Cleopatra ${ }^{48}$ de Thomas Francis
Dicksee (1819-1895) ${ }^{49}$ est assise, s'appuyant sur l'accoudoir droit d'un fauteuil à haut dossier. Parée de quelques bijoux, elle a la tête penchée vers la gauche, le regard fixé vers le bas. Ses cheveux lui tombent le long du cou sur les épaules, encadrant son collier et sa boucle d'oreille. Dans sa main droite elle tient un serpent, sorti d'un panier plein de feuilles qui est posé sur une petite table, couverte d'un joli tissu rouge. Sa blouse blanche est bouclée par quelques perles sur les épaules et les bras. Sa jupe de satin gris tombe sur ses pieds nus qui reposent sur un coussin. Le sol est recouvert d'une grande peau de léopard. Sur un petit escalier, à droite dans le tableau, figure un candélabre dont l'ombre tombe sur un mur décoré de fleurs selon la mode égyptienne.

III.C.1.b. Dans Cleopatra's last hour ${ }^{50}$ attribué à Dicksee (1819-1895), la reine, tout comme celle de Delacroix ${ }^{51}$, est parée de nombreux bijoux et regarde devant soi, profondément plongée dans des réflexions moroses. L'absence du paysan attire toute l'attention du spectateur sur sa personne. D'un panier en rotin, posé sur l'accoudoir de son fauteuil, sortent quelques feuilles de vigne et la tête du serpent qui causera sa mort.

Le bijou le plus remarquable est un petit serpent, un soleil (?) sur la tête, enroulé autour de son bras gauche. Le bleu de certaines perles contraste avec le tissu rouge brodé sur lequel repose son bras. Une espèce de chiton, dont les coutures sont nouées par une série de perles, enveloppe en partie son corps. L'ensemble

43. Griffiths 1961: 116.

44. Daix 1960: 286.

45. Griffiths 1961: 116 ; Chauveau 1998: 106-07.

46. von Wertheimer 1935: 312 .

47. Plutarque XIII. 86.6 ; von Wertheimer 1935: 312 ; Daix 1960: 285.

48. D'après Bénézit, vol. 4, 1999: 557, la Cleopatra de Dicksee, 1862, 35,6 x 30,5, aurait été présentée à une vente à Londres le 9 février 1990.

49. Né à Londres le 13 décembre 1819, mort dans sa ville natale le 6 novembre 1895 .

50. 1868 ; huile sur toile ; $76,8 \times 63,5 \mathrm{~cm}$; Sotheby’s Londres, 12 avril 1985, n 156 ill.

51. Ritschard / Morehead 2004: 251 fig. 1. 
de ce tableau se rapproche de l'autre Cleopatra de Dicksee (III.C.1.a), dont le visage est cependant plus dur et beaucoup moins fin.

III.C.1.c. La très belle peinture The death of Cleopatra $^{52}$ de Reginald Arthur ${ }^{53}$ présente une mourante, étendue sur son lit, sa tête jetée en arrière. Sa main gauche se crispe sur les draps, celle de droite enserre le serpent, dont son regard s'éloigne. Son pied gauche est courbé, les orteils en crampe. En partie couverte d'un tissu très fin, très léger, la mourante est ornée de bijoux qui indiquent sa royauté: la couronne, le collier, les bagues et le bracelet où on reconnaît les plumes et les pattes d'un oiseau aux ailes éployées. À côté d'elle, sur le pied du lit, gît sa servante Iras. Une troisième figure, à peine visible, se remarque en haut entre deux colonnes, au-dessus d'une faible fumée bleu pâle, émanée d'une lampe à huile prête à s'éteindre. Il s'agit peut-être d'une statue. Tout le décor est exécuté dans un style égyptisant. Un passage à gauche est garni d'une gorge égyptienne et d'un tore, tandis que deux fines colonnettes, de chaque côté du lit, sont décorées de pétales de lotus. La petite table, sur laquelle est posée la corbeille, pleine de feuilles de figues, est décorée d'un pharaon ailé, uræus au front. Le tore qui sort en partie d'en dessous des draps, se présente avec des stries transversales et obliques représentant des rubans. Une peau de léopard, suspendue derrière la tête de Cléopâtre, cache une statue, probablement un sphinx ou un lion, dont la patte est visible en haut du lit. Le contraste en[142 ] tre la blancheur de Cléopâtre et les tons sombres des pièces voisines, ainsi que l'emploi des couleurs complémentaires, le rouge et le vert, le bleu et l'orange, contribuent grandement à la beauté de ce tableau.
III.C.1.d. La Kleopatra ${ }^{54}$ d'Emil Teschendorff(1833-1894) $)^{55}$ montre à plusieurs égards une certaine ressemblance avec la Cleopatra de Dicksee (3a): les cheveux noirs et longs, les rares bijoux, le serpent dans la main droite, la robe satinée, la peau de léopard par terre. La reine est à moitié allongée sur son fauteuil, accoudée sur son côté gauche. Un panier en rotin plein de roses, est posé à côté de son bras droit. D'un autre, tombé par terre, à côté de son pied droit, est sorti le serpent, qu'elle enserre de sa main droite. Ses yeux ouverts sont fixés vers le bas. Une servante tourne le dos à la scène, s'éloignant vers la gauche. Une espèce de baldaquin et une grande draperie suspendue derrière la reine mourante forment le décor très sombre du tableau.

III.C.1.e. Dans La mort de Cléopâtre ${ }^{56}$ signé "Paul Bouchard/1887" (1853-1937) $)^{57}$, le peintre s'écarte résolument des sentiers battus en faisant fi du texte de Plutarque et en donnant libre cours à sa propre imagination. Dans une sobre palette brune, il situe la fin de Cléopâtre dans un intérieur totalement arabe, comme l'indiquent le réseau d'entrelacs en haut à gauche, les panneaux ornés de versets du Coran et la décoration du sofa. La reine, allongée par terre, sa tête reposant sur le sofa, son petit corsage déboutonné, est morte et attire des spectateurs noirs, entrant dans la pièce. Elle porte une petite couronne sans uræus et des anneaux autour des bras et des pieds. Trois servantes s'activent dans la partie gauche du tableau. Il semblerait que Bouchard n'ait point cru que la mort de Cléopâtre ait été provoquée par la morsure d'un aspic mais qu'il l'ait plutôt attribuée à un poison qu'elle aurait avalé par la bouche.

52. 1892 ; huile sur toile ; collection particulière. Voir Humbert 1989: 247 ill.

53. 1881: première fois mentionné ; 1896: dernière fois mentionné. Voir Saur 1999, vol. 1: 421.

54. Date inconnue ; huile sur toile ; Chur (Suisse), Rätisches Kunstmuseum. Voir Müller-Fulda 2004: 6-16 ill.

55. Né à Stettin le 15 mai 1833, mort à Berlin le 4 juin 1894.

56. Huile sur toile ; 274,3 x 365,5 cm ; Christie's New York, 14 octobre 1993, n 58 ill.

57. Né à Paris en 1853, mort dans sa ville natale en 1937. 
Un gobelet renversé tout près de ses genoux confirme cette impression.

III.C.1.f. Après l'exposition de son tableau Cléopâtre reine d'Égypte au tombeau $d^{\prime}$ 'Antoine ${ }^{58}$ au Salon de 1895 à Paris, LouisMarie Baader (1828-1919) peint vers 1899 La mort de Cléopâtre, reine d'Égypte ${ }^{59}$. À gauche entre un Romain en toge blanche. Il trouve la reine apparemment morte ou mourante, Iras, sa femme de chambre et coiffeuse, agonisante sur le tapis rouge, et Charmion, agenouillée à côté du canapé, regardant la moribonde. Les motifs égyptisants abondent dans ce merveilleux tableau : les colonnes, le décor derrière le canapé, le cobra, la harpe à côté du tapis, le mur où passe le Romain, l'éventail aux plumes d'autruche contre le dossier du sofa et le bas du décor juste à côté. L'élément architectural à droite dans le tableau est garni de deux têtes de lion, décoration peut-être empruntée à Prisse d'Avennes ${ }^{60}$, qui dessine ce même élément avec trois têtes de lion. Une même architecture avec une tête d'Hathor figure dans le tableau de Baader cité ci-dessus ${ }^{61}$. On notera en particulier un pittoresque détail qu'on retrouve aussi dans les tableaux de Cabanel, de Collier et de Makart ${ }^{62}$, à savoir un chasse-mouches ou un éventail porté par une servante ou simplement posé par terre. La finesse de ses plumes et ses couleurs vives attirent l'attention. On constate que dans la peinture de Baader la reine égyptienne est dépourvue de ses plus beaux atours; curieusement, elle ressemble davantage à une petite paysanne. La beauté de l'œuvre réside avant tout dans le flamboyant décor égyptisant dans lequel le peintre situe la scène.

\section{Tableaux déjà répertoriés}

III.C.2.a. Charles-Pompée Boulanger de Boisfremont (1773-1838): La mort de Cléopâtre (Ritschard / Morehead 2004: 328, 329 ill.).

III.C.2.b. Raymond Auguste Quinsac Monvoisin (1794-1870): La mort de Cléopâtre esquisse huile sur papier contrecollé sur toile (Ritschard / Morehead 2004: 326, 327 ill.).

III.C.2.c. Eugène Delacroix (1798-1863): Cléopâtre et le paysan (Ritschard / Morehead 2004: 251 fig. 1, 252-253, 255).

III.C.2.d. German von Bohn (1812-1899): Der Tod der Kleopatra (De Meulenaere 1992: 107 ill.).

III.C.2.d. Gustave Lassalle-Bordes (1814-1848): La mort de Cléopâtre (De Meulenaere 1992: 100 ill.).

III.C.2.e. Arnold Böcklin (1827-1901): Der Tod der Kleopatra, 1872 (Ritschard / Morehead 2004: 345, 347 ill.).

III.C.2.f. Arnold Böcklin (1827-1901): Der Tod der Kleopatra, 1878 (Ritschard / Morehead 2004: 345 fig. 1).

III.C.2.g. Henri-Blaise Dejussieu (1828-1882): Cléopâtre (De Meulenaere 1992: 100 ill.; Ritschard / Morehead 2004: 335 ill.).

III.C.2.h. Valentine Cameron Prinsep (1838-1904): The Death of Cleopatra (De Meulenaere 1992: 104-105 ill.).

III.C.2.i. Hans Makart (1840-1884): Der Tod

58. Voir plus haut note 33 .

59. Vers 1899; huile sur toile ; 99 x 125 cm ; Rennes, Musée des Beaux-Arts, Inv. 20.751. Voir Flamarion 1993: 142 ill. ; Murat 2003: 47 fig. 12 ill.

60. Prisse D'Avennes, 1878: pl. XX-6.

61. Voir II.C.1.c.

62. Cabanel: De Meulenaere 1992: 99 ; Ritschard / Morehead 2004: 245, 261; Collier: Ritschard / Morehead 2004: 344 ; Makart: De Meulenaere 1992: 100 ; Ritschard / Morehead 2004: 348. 
der Kleopatra (Ritschard / Morehead 2004: 348 fig. 1; De Meulenaere 1992: 100 ill.).

III.C.2.j. Jean André Rixens (1846-1924): La mort de Cléopâtre (De Meulenaere 1992: 102-103 ill.).

III.C.2.k. John Collier (1850-1934): The Death of Cleopatra (Ritschard / Morehead 2004: 344 fig. 1).

\section{Les funérailles de Cléopâtre}

César, tout fâché qu'il était de la mort de cette femme, admira sa grandeur d'âme, et la fit ensevelir avec une magnificence royale auprès d'Antoine. Il fit faire aussi à ses suivantes des obsèques honorables.

(Plu., Ant. 86. 7)
Après les funérailles solennelles de Cléopâtre, Octave vint s'emparer du trésor enfermé dans le mausolée. À cause de celui-ci, les taux d'intérêts baissèrent de 12 à $4 \%$ à Rome ${ }^{63}$.

\section{Tableau déjà répertorié}

III.D.2.a. Frederick Arthur Bridgman (1847-1928): Cleopatra's Funeral Barge (De Meulenaere 1992: 107 ill.; Ritschard / Morehead 2004: 356 fig. 1 ill.).

Par sa vie tumultueuse, par sa fin tragique, surtout, Cléopâtre est entrée dans la légende. Une légende qui en a fait la plus célèbre reine d'Égypte, éclipsant toutes celles qui l'ont précédée. L'hommage posthume que lui ont rendu les peintres au travers des siècles a largement contribué à l'auréoler d'une gloire immortelle. 


\section{Bibliographie}

ACKerman, G.M.

1986 La vie et l'œuvre de Jean-Léon Gérôme. Paris.

BALDWIN, B.

1964 The Death of Cleopatra VII, JEA 50: 181-182.

BÉNÉZIT, E.

1999 Dictionnaire des peintres, sculpteurs, dessinateurs et graveurs. Paris, réimpr. en 14 vols.

BERKo, P. \& V.

1981 Dictionnaire des peintres belges nés entre 1750 et 1875 . Bruxelles/Knokke-Zoute.

Bingen, J.

1996 Cléopâtre: L'image et le diadème, Bulletin de la Classe des Lettres et des Sciences Morales et Politiques. Académie Royale de Belgique $6^{\mathrm{e}}$ série, VII, 1-6: 235-248.

Chauveau, $\mathrm{M}$.

1997 L'Égypte au temps de Cléopâtre. Paris. 1998 Cléopâtre au-delà du mythe. Paris.

DAIX, P.

1960 Cléopâtre. Les femmes célèbres de l'histoire. Paris.

De Meulenaere, $\mathrm{H}$.

1992 L'Égypte ancienne dans la peinture $d u$ XIXe siècle. Knokke.

Dobias, $\mathrm{H}$.

$1934 \mathrm{La}$ donation d'Antoine à Cléopâtre en l'an 34 av. J.-C., Annuaire de l'Institut de Philologie et d'Histoire Orientales II: 287-314.

Foreman, L.

2000 La légende engloutie de Cléopâtre. Grenoble.
Foss, M.

1997 The Search for Cleopatra. New York.

Flamarion, E.

1993 Cléopâtre, vie et mort d'un pharaon. $\mathrm{Pa}-$ ris.

Goudchaux, G.W.

1992 Archibios. Sauveur des " effigies » de Kléopâtre VII, Atti del Sesto Congresso Internazionale di Egittologia I, Turin: 651-656.

GreEnaCRe, Fr.

1988 Francis Danby 1793-1861. The Tate Gallery in Association with the City of Bristol Museum and Art Gallery. (Cat. d'exposition Bristol et Tate Gallery). Balding.

GRIFFITHS, J.G.

1961 The Death of Cleopatra VII, JEA 47: 113-118.

GRIMAL, N.

1998 La gloire d'Alexandrie. (Cat. d'exposition Paris 1998). Paris.

Hess, $\mathrm{H}$.

1949 William Etty. Centenary Exhibition. (Cat. d'exposition Walker Art Gallery York), York.

HöLBL, G.

1994 Geschichte des Ptolemäerreichs. Politik, Ideologie und religiöse Kultur von Alexander dem Grossen bis zur römischen Eroberung. Darmstadt.

Hughes-Hallett, L.

1990 Cleopatra. Histories, Dreams and Distortions. London.

HUMBert, J.-M.

1989 L'Égyptomanie dans l'art occidental. Paris. 
Humbert, J.-M.; Pantazzi, M.; Ziegler, Chr.

1994 Egyptomania. L'Égyptedans l'artoccidental 1730-1930. (Cat. d'exposition Paris). Paris.

Huss, W.

2001 Ägypten in hellenistischer Zeit 332-30v. Chr. Munich.

JULER, C.

1987 Les orientalistes de l'école italienne. Paris.

Lacovara, P.; Teasly Trope, B.; D’Auria, S.H.

2001 The Connector's Eye: Masterpieces of Egyptian Art from the Thalassic Collection, Ltd. (Cat. d'exposition Atlanta). Atlanta.

LE ROBERT

1975 Dictionnaire universel de la peinture. Paris, 6 vols.

MüLler-Fulda, A.B.

2004 Ein Leben zwischen Orient und Okzident - die Familie von Planta im 19. Jahrhundert, Die Mumie der Ta-di-Isis. Eine Reise vom Nil zum Rhein. Chur (Suisse).

Murat, P.

2003 Cléopâtre, la légende vivante d'une reine morte. (Cat. d'exposition Nîmes). Nîmes.

Prisse D'Avennes, A.C.Th.E.

1878 Histoire de l'Art Égyptien d'après les monuments. Atlas I. Paris.

Ritschard, Cl.; Morehead A.

2004 Cléopâtre dans le miroir de l'art occidental. (Cat. d'exposition Genève). Genève.

[146]

S.A.

1981 J.-L. Gérôme 1824-1904. Peintre, sculpteur et graveur. Ses æuvres conservées dans les collections françaises publiques et privées. (Cat. d'exposition Vesoul). Vesoul.

SARTRE, M.

1999 Portrait d'une inconnue, L'Histoire 238 (décembre): 32-34.
SAUR, K.G.

1999-2000 Allgemeines Künstlerlexikon. Bio-Bibliographischer Index. Munich/ Leipzig, 10 vols.

SkeAt, T.C.

1953 The last Days of Cleopatra, The Jourrnal of Roman Studies 43: 98-100.

Tarn, W.W.; Charlesworth, M.P.

1965 Octavian, Anthony and Cleopatra. Cambridge.

VOLKMANN, H.

1953 Kleopatra. Munich.

VON WERTHEIMER, O.

1935 Cléopâtre, reine des rois. Paris.

WHITEHORNE, J.

1994 Cleopatras. London \& New York.

ZiEgler, Chr.

1994 Les jours et les nuits de Cléopâtre, Muséart 37 (février): 52-55.

\section{Traductions}

Dion Cassius

Histoire Romaine VII. Traduit par E. Gros. Editeur: Firmin Didot. Paris, 1865.

Pline L'Ancien

Histoire Naturelle. Livre IX. Traduit par E. de Saint-Denis. Edition « Les Belles Lettres ». Paris, 1955.

Plutarque

Vies IX. Alexandre - César. Traduit par R. Flacelière et E. Chambry. Edition « Les Belles Lettres ». Paris, 1975. Vies XIII . Démétrios - Antoine. Traduit par R. Flacelière et E. Chambry. Edition « Les Belles Lettres ». Paris, 1977.

SUÉTONE

Vies des douze Césars. I César - Auguste. Traduit par H. Ailloud. Edition « Les Belles Lettres ». Paris, 1931. 


\section{Juan Antonio Belmonte}

Instituto de Astrofísica de Canarias

Vía Láctea $\mathrm{S} / \mathrm{N}$

38200 La Laguna, Tenerife - Islas Canarias

España

$$
\text { jba@iac.es }
$$

\section{Marcelo Campagno}

Instituto Multidisciplinario de Historia y Ciencias Humanas

Departamento de Egiptología

Saavedra $15,5^{\circ}$ piso

C1083ACA Buenos Aires

Argentina

mcampagno@fibertel.com.ar

\section{Lucía Elena Díaz-Iglesias Llanos}

Dpto. de Prehistoria, Antropología e Historia Antigua

Facultad de Geografía e Historia - Campus de Guajara

38071 Universidad de La Laguna

Tenerife - Islas Canarias

España

luch68@hotmail.com

\section{Magdi Fekri}

Faculty of Tourism

Minufiya University

El-Sadat City

Egypt

per-ankh@hotmail.com

\section{Cristina Pino Fernández}

cristinapino@telefonica.net 


\section{Mosalam Shaltout}

Helwan Observatory

Helwan, Cairo

Egypt

mosalamshaltout@hotmail.com

\section{Eric P. Uphill}

Institute of Archaeology

University of London

31-34 Gordon Square

London WC1H OPY

United Kingdom

\section{Marie-Paule Vanlathem}

Association Égyptologique Reine Élisabeth Musées Royaux d'Art et d'Histoire

Parc du Cinquantenaire 10

B-1000 Bruxelles

Belgique

mariepaule.vanlathem@belgacom.net 


\section{Trabajos de Egiptología}

\section{Papers on Ancient Egypt}

P

Número 4 2005 


\title{
Consejo Editorial
}

\author{
Miguel Á. Molinero Polo \\ Universidad de La Laguna \\ Antonio Pérez Largacha \\ Universidad de Castilla-La Mancha \\ José-R. Pérez-Accino \\ Birkbeck, Universidad de Londres \\ Covadonga Sevilla Cueva \\ Universidad Autónoma de Madrid
}

\section{Comité Científico}

Josep Cervelló i Autuori

Aula Aegyptiaca, Barcelona

Ma José López Grande

Universidad Autónoma de Madrid

Josep Padró i Parcerisa

Universitat de Barcelona

$\mathrm{M}^{\mathrm{a}}$ Carmen Pérez Die

Museo Arqueológico Nacional, Madrid

Ester Pons Mellado

Museo Arqueológico Nacional, Madrid

José M. Serrano Delgado

Universidad de Sevilla

\section{Colaboradores Editoriales}

Linda Steynor

English editorial assistant

Hervé Mouriacoux

Assistant editoriale pour langue française 
Juan Antonio Belmonte, Mosalam Shaltout, Magdi Fekri

Astronomy and landscape in Ancient Egypt:

Challenging the enigma of the minor step pyramids

\section{Marcelo Campagno}

Two observations on the tales of The contendings of Horus and Seth and Truth and Falsehood

\section{Lucía Elena Díaz-Iglesias Llanos}

Commentary on Heracleopolis Magna from the theological perspective (I):

The image of the local lakes in the vignette of chapter 17 of the Book of the Dead

Cristina Pino Fernández

Prince Ahmose Sapair again

Eric P. Uphill

Irrigation basins and cultivated land under the Twelfth Dynasty

\section{Marie-Paule Vanlathem}

Cléopâtre dans le miroir de la peinture du XIXe siècle 
Trabajos de Egiptología está producida por Isfet. Egiptología e Historia.

c/ Blanco $1,2^{\circ}$

38400 Puerto de la Cruz

Tenerife - Islas Canarias

España

Maquetación: Ignacio Cases

(C) Autores de los artículos aparecidos

y Consejo Editorial de Trabajos de Egiptología - Papers on ancient Egypt

Depósito legal:

ISSN 1695-4750

Impresión: 